\title{
A NOTE ON THE VARIATIONAL STRUCTURE OF AN ELLIPTIC SYSTEM INVOLVING CRITICAL SOBOLEV EXPONENT
}

\section{MARIO ZULUAGA}

Received 22 August 2002 and in revised form 10 January 2003

We consider an elliptic system involving critical growth conditions. We develop a technique of variational methods for elliptic systems. Using the well-known results of maximum principle for systems developed by Fleckinger et al. (1995), we can find positive solutions. Also, we generalize the systems results obtained (for the scalar case) by Brézis and Nirenberg (1983). Also, we give applications to biharmonic equations.

\section{Introduction}

In this paper, we are concerned with the existence of solutions of the elliptic system

$$
\begin{aligned}
& -\Delta u=\lambda u+\delta v+g_{1}(u, v), \\
& -\Delta v=\theta u+\gamma v+g_{2}(u, v)
\end{aligned}
$$

on $\Omega$ and $u=v=0$ on $\partial \Omega$, where $\Omega \subset \mathbb{R}^{n}, n>2$, is a bounded domain with the smooth boundary $\partial \Omega, \lambda, \delta, \theta$, and $\gamma$ are real numbers, and $g_{1}$, $g_{2}$ are real-valued functions with critical growth.

The purpose of this paper is to extend the results, obtained in [4], of elliptic equations for the case of only one equation (the scalar case) to the case of elliptic systems as (1.1). Our main tools are a variational approach developed for functionals with values on $\mathbb{R}^{2}$ (we want to remark that it is an important innovation in this paper), a maximum principle for systems developed in [6], and a minimax approach as in [1]. 
Letting

$$
\begin{aligned}
& U=(u, v), \quad-\vec{\Delta} U=(-\Delta u,-\Delta v), \\
& \mathbf{A}=\left(\begin{array}{ll}
\lambda & \delta \\
\theta & \gamma
\end{array}\right), \quad G(U)=\left(g_{1}(u, v), g_{2}(u, v)\right),
\end{aligned}
$$

we can write (1.1) as

$$
-\vec{\Delta} U=\mathbf{A}(U)+G(U), \quad U=\Theta=(0,0)
$$

on $\Omega$ and $U=\Theta$ on $\partial \Omega$.

It is not common to find in the literature a variational approach of problems like (1.1). Our starting point is [5] where resonance cases were considered. In that paper, the authors considered the functional

$$
J_{ \pm}(U)=\frac{1}{2} \int_{\Omega}\left[|\nabla u|^{2} \pm|\nabla v|^{2}-\left(\lambda u^{2} \pm 2 \delta u v+\gamma v^{2}\right)\right]-\int_{\Omega} F(U),
$$

where $\nabla F=\left(g_{1}, g_{2}\right)$, for the study problem (1.1) in the cases

$$
\mathbf{A}=\left(\begin{array}{ll}
\lambda & \delta \\
\delta & \gamma
\end{array}\right), \quad \mathbf{A}=\left(\begin{array}{cc}
\lambda & -\delta \\
\delta & \gamma
\end{array}\right)
$$

The first case is known as cooperative problem and the second one as noncooperative problem. It is important to remark that $J_{ \pm}$are real-valued functionals and thus it is no clear how critical points of $J_{ \pm}$, called weak solutions in that paper, became classical solutions of (1.3). So, it is necessary to maintain the classical concept of weak solutions extended now to systems like (1.1) and then to develop a critical point theory for functionals with values on $\mathbb{R}^{2}$.

Weak solutions of (1.1)

It is natural to define weak solutions of (1.1) as follows: $u, v \in H_{0}^{1}(\Omega)$ are weak solutions of (1.1) if, for all $\phi \in H_{0}^{1}(\Omega)$,

$$
\begin{aligned}
& \int_{\Omega} \nabla u \nabla \phi-\lambda u \phi-\delta v \phi-g_{1}(u, v) \phi=0, \\
& \int_{\Omega} \nabla v \nabla \phi-\theta u \phi-\gamma v \phi-g_{2}(u, v) \phi=0 .
\end{aligned}
$$

The novelty here is that we can choose a functional whose critical points are weak solutions of (1.1) in the sense of (1.6). In Section 3, we 
present such a functional. Also, we can use the regularity theory to show that weak solutions of (1.1) are classical solutions as well.

Our paper mainly focuses on the cases $g_{1}(u, v)=|u|^{(n+2) /(n-2)}$ and $g_{2}(u$, $v)=|v|^{(n+2) /(n-2)}, n>2$, and as in [4] it is necessary to distinguish between $n=3$ and $n \geq 4$ cases.

A superlinear case was considered in [9] where $g_{1}(u, v)=|u|^{r}$ and $g_{2}(u, v)=|v|^{r}, r<(n+2) /(n-2)$; sufficient conditions were given in that paper for the existence of positive solutions. The techniques used there was Leray-Schauder degree theory and measure theory.

\section{Preliminaries and notation}

We define $\mathbf{L}^{r}(\Omega)=L^{r}(\Omega) \times L^{r}(\Omega), r \geq 1$, and the following operations: for all $U=(u, v)$ and $\Phi=(\phi, \psi)$, we have

(1) $U * \Phi=(u \phi, v \psi)$;

(2) $-\vec{\Delta} U=(-\Delta u,-\Delta v)$;

(3) $U^{p}=\left(u^{p}, v^{p}\right)$;

(4) $D_{i} U=\left(D_{i} u, D_{i} v\right)$;

(5) $|\nabla U|^{2}=\left(|\nabla u|^{2},|\nabla v|^{2}\right)$;

(6) $|U|=(|u|,|v|)$;

(7) $U \geq \Theta=(0,0)$ if and only if $u \geq 0$ and $v \geq 0$. Also, $U>\Theta$ if and only if $u \geq 0$ and $v \geq 0$, and $u>0$ or $v>0$;

(8) $\alpha U=(\alpha u, \alpha v)$ for $\alpha \in \mathbb{R}$;

(9) $\vec{\alpha} * U=(a u, b v)$ for all $\vec{\alpha}=(a, b) \in \mathbb{R}^{2}$;

(10) \|\|$U \mid \|_{r}=\left(\|u\|_{r},\|v\|_{r}\right) \in \mathbb{R}^{2}$ for all $U \in \mathbf{L}^{r}(\Omega), r \geq 1$;

(11) $\int_{\Omega} U=\left(\int_{\Omega} u, \int_{\Omega} v\right)$.

\section{Pohozaev's identity}

Consider the general elliptic system

$$
-\vec{\Delta} U=G(U), \quad U=\Theta
$$

on $\Omega$ and $U=\Theta$ on $\partial \Omega$, where $G(U)=\left(g_{1}(u, v), g_{2}(u, v)\right)$. Then we define

$$
F(U)=\int_{\Theta}^{u} G(t) d t=\left(\int_{0}^{u} g_{1}(t, v) d t, \int_{0}^{v} g_{2}(u, t) d t\right) .
$$

As in the scalar case, if $U$ is a smooth function satisfying (2.1), then it is easy to check that

$$
n \int_{\Omega} F(U)+\frac{2-n}{2} \int_{\Omega} U * G(U)=\frac{1}{2} \int_{\partial \Omega}\langle X, \vec{\eta}\rangle\left(\frac{\partial U}{\partial \vec{\eta}}\right)^{2},
$$

where $\partial \vec{\eta}$ denotes the normal outer vector on $\partial \Omega$. 
Particular cases

(a) If, for example, $G(U)=|U|^{(n+2) /(n-2)}, n>2$, we see that

$$
\begin{gathered}
n \int_{\Omega} F(U)=\frac{n}{2^{*}} \int_{\Omega}|U|^{2^{*}}, \\
\frac{2-n}{2} \int_{\Omega} U * G(U)=\frac{2-n}{2} \int_{\Omega}|U|^{2^{*}},
\end{gathered}
$$

where $2^{*}=2 n /(n-2)$.

Then, if $\Omega$ is starshaped $(\langle X, \vec{\eta}\rangle>0)$ by (2.3), we conclude that there is no positive solution of (2.1). This result is well known from 1965, see [8].

(b) If, for example, $G(U)=\mathbf{A}(U)+|U|^{(n+2) /(n-2)}, n>2$, Pohozaev's identity tells us that

$$
\int_{\Omega}(\lambda, \gamma) * U^{2}+\frac{n+2}{2} \int_{\Omega}(\delta, \theta) * U * \tilde{U}=\frac{1}{2} \int_{\partial \Omega}\langle X, \vec{\eta}\rangle\left(\frac{\partial U}{\partial \vec{\eta}}\right)^{2}
$$

where $\tilde{U}=(v, u) \in \mathbf{H}_{0}^{1}(\Omega)$ and $\mathbf{H}_{0}^{1}(\Omega)=H_{0}^{1}(\Omega) \times H_{0}^{1}(\Omega)$. Identity $(2.5)$ gives us the following negative result.

THEOREM 2.1. Suppose that $\Omega$ is starshaped and that $\lambda, \delta, \theta, \gamma \leq 0$ or also $(\delta, \theta) \geq \Theta$ and $(\lambda, \gamma) \geq\left(\lambda_{1}, \lambda_{1}\right)$, where $\lambda_{1}$ is the first eigenvalue of $-\Delta$, then there is no positive solution for problem (1.1) for the case $g_{1}(u, v)=|u|^{(n+2) /(n-2)}$ and $g_{2}(u, v)=|v|^{(n+2) /(n-2)}$.

Proof. For the first case, it is only sufficient to observe that if $U$ is a solution of (1.1), then $U * \tilde{U}>\Theta$, which is contradictory with (2.5). In the second case, if $U>\Theta$ is a solution of

$$
\begin{gathered}
-\vec{\Delta} U=\mathbf{A}(U)+|U|^{(n+2) /(n-2)} \quad \text { on } \Omega, \\
U=\Theta \quad \text { on } \partial \Omega,
\end{gathered}
$$

then

$$
\int_{\Omega}(-\vec{\Delta} U) * \Phi_{1}=\int_{\Omega} \mathbf{A}(U) * \Phi_{1}+\int_{\Omega}|U|^{(n+2) /(n-2)} * \Phi_{1}
$$

where $\Phi_{1}=\left(\phi_{1}, \phi_{1}\right)$ and $\phi_{1}$ is the first eigenfunction of $-\Delta$. Then we have

$$
\begin{aligned}
& \int_{\Omega}\left(\lambda_{1}, \lambda_{1}\right) * U * \Phi_{1} \\
& \quad=\int_{\Omega}(\lambda, \gamma) * U * \Phi_{1}+\int_{\Omega}(\delta, \theta) * \tilde{U} * \Phi_{1}+\int_{\Omega}|U|^{(n+2) /(n-2)} * \Phi_{1} .
\end{aligned}
$$


Since $\int_{\Omega}(\delta, \theta) * \tilde{U} * \Phi_{1} \geq \Theta$, the foregoing identity tells us that $U=\Theta$, contrary to our hypothesis $U>\Theta$. This theorem extends to elliptic systems, a well-known result of [4].

Now, we will borrow the ideas of a maximum principle developed in [6] to prove the following theorem.

Theorem 2.2. If $0<\lambda, \gamma<\lambda_{1},(\delta, \theta) \geq \Theta$, and $\operatorname{det}\left(\lambda_{1} \mathbf{I}-\mathbf{A}\right)>0$, then all nonzero solutions of

$$
\begin{aligned}
& -\Delta u=\lambda u+\delta v+|u|^{(n+2) /(n-2)} \\
& -\Delta v=\theta u+\gamma v+|v|^{(n+2) /(n-2)}
\end{aligned}
$$

on $\Omega$ and $u=v=0$ on $\partial \Omega$ are no negative solutions.

Proof. In this proof, we use the arguments of maximum principle for systems developed in [6], which plays a crucial role in the proof of Theorem 3.1. Suppose that $(u, v)=U \in \mathbf{H}_{0}^{1}(\Omega)$ is a nonzero solution of foregoing system. Let $\Phi=(\phi, \psi) \in \mathbf{H}_{0}^{1}(\Omega)$ such that

$$
\Phi=\max \{\Theta,-U\}
$$

If we multiply $-\vec{\Delta} U=\mathbf{A}(U)+|U|^{(n+2) /(n-2)}$ by $\Phi$, we get

$$
\begin{aligned}
-\int_{\Omega} \vec{\Delta} U * \Phi & =\int_{\Omega} \nabla U * \nabla \Phi=-\int_{\Omega}|\nabla \Phi|^{2} \\
& =-\int_{\Omega}(\lambda, \gamma) *|\Phi|^{2}+\int_{\Omega}(\delta, \theta) * \tilde{U} * \Phi+\int_{\Omega}|U|^{(n+2) /(n-2)} * \Phi,
\end{aligned}
$$

which produces

$$
\int_{\Omega}\left(\lambda_{1}, \lambda_{1}\right) *|\Phi|^{2} \leq \int_{\Omega}|\nabla \Phi|^{2} \leq \int_{\Omega}(\lambda, \gamma) *|\Phi|^{2}+\int_{\Omega}(\delta, \theta) * \widetilde{\Phi} * \Phi
$$

and then

$$
\int_{\Omega}\left(\lambda_{1}-\lambda, \lambda_{1}-\gamma\right) *|\Phi|^{2} \leq \int_{\Omega}(\delta, \theta) * \widetilde{\Phi} * \Phi
$$


232 A note on the variational structure

From (2.13) and Cauchy-Schwarz inequality, we conclude that

$$
\begin{aligned}
& \left(\lambda_{1}-\lambda\right)\|\phi\|_{L^{2}}^{2} \leq \delta\|\phi\|_{L^{2}}\|\psi\|_{L^{2}}, \\
& \left(\lambda_{1}-\gamma\right)\|\psi\|_{L^{2}}^{2} \leq \theta\|\phi\|_{L^{2}}\|\psi\|_{L^{2}},
\end{aligned}
$$

then

$$
\operatorname{det}\left(\lambda_{1} \mathbf{I}-\mathbf{A}\right)\|\phi\|_{L^{2}}^{2}\|\psi\|_{L^{2}}^{2} \leq 0
$$

Inequality (2.15) implies that $\phi=0$ or $\psi=0$ and then $\phi=0$ and $\psi=0$. By regularity, we conclude that $U \geq \Theta$.

Also, for weakly coupled cooperative elliptic systems, a maximum and strong maximum principle and its characterization have been developed by López-Gómez and Molina-Meyer in [7, Theorems 2.1 and 2.6] which can be used in Theorem 2.2 in the cooperative case.

\section{Main results}

For all $U=(u, v), \Phi=(\varphi, \psi) \in \mathbf{L}^{2}(\Omega)$. We define an $\mathbb{R}^{2}$ inner product with the following bracket:

$$
[U, \Phi]=\left(\int_{\Omega} u \varphi, \int_{\Omega} v \psi\right) \in \mathbb{R}^{2}
$$

It is easy to check that

(1) $[U, \Phi]=[\Phi, U]$ for any $U, \Phi \in \mathrm{L}^{2}(\Omega)$;

(2) $[U, \Phi+\Lambda]=[U, \Phi]+[U, \Lambda]$ for any $U, \Phi, \Lambda \in \mathrm{L}^{2}(\Omega)$;

(3) $[\lambda U, \Phi]=\lambda[U, \Phi]=\left(\int_{\Omega} \lambda u \varphi, \int_{\Omega} \lambda v \psi\right)$ for any $\lambda \in \mathbb{R}$ and $U, \Phi \in \mathbf{L}^{2}(\Omega)$;

(4) $\left[(\vec{\Delta})^{-1} U, \Phi\right]=\left[U,(\vec{\Delta})^{-1} \Phi\right]$ for any $U, \Phi \in \mathrm{L}^{2}(\Omega)$.

The weak solutions of our problem (1.1), like we have defined in (1.6), can be represented as critical points of functional $\mathbf{J}: \mathbf{H}_{0}^{1}(\Omega) \rightarrow \mathbb{R}^{2}$, defined as

$$
\mathbf{J}(U)=\frac{1}{2}[\nabla U, \nabla U]-\frac{1}{2}[\mathbf{C U}, U]-\int_{\Omega} F(U),
$$

where $F(U)=\int_{\Theta}^{U} G(t) d t=\left(\int_{0}^{u} g_{1}(t, v) d t, \int_{0}^{v} g_{2}(u, t) d t\right), U=(u, v) \in \mathbf{H}_{0}^{1}(\Omega)$, and

$$
\mathbf{C}=\left(\begin{array}{cc}
\lambda-\delta & 2 \delta \\
2 \theta & \gamma-\theta
\end{array}\right)
$$


In fact, a calculation shows that for $U=(u, v)$ and $\Phi=(\phi, \psi)$,

$$
\begin{aligned}
\mathbf{J}^{\prime}(U)(\Phi) & =\left.\frac{d}{d t} \mathbf{J}(U+t * \Phi)\right|_{t=\Theta} \\
& =[\nabla U, \nabla \Phi]-\frac{1}{2}\{[C U, \Phi]+[C \Phi, U]\}-[G(U), \Phi] .
\end{aligned}
$$

Now, in the case $\Phi=(\phi, \phi)$, the previous equality is transformed in

$$
\mathbf{J}^{\prime}(U)(\Phi)=[\nabla U, \nabla \Phi]-[\mathbf{A} U, \Phi]-[G(U), \Phi],
$$

then critical points of $\mathbf{J}$ become weak solutions of (1.1).

For our main theorem, we use a Lagrange multiplier method which has been adapted to our purpose. Let

$$
\begin{aligned}
\overrightarrow{\mathbf{s}}_{\mathrm{C}} & =\inf _{\|U\|_{p+1}=\overrightarrow{\mathbf{1}}}\{[\nabla U, \nabla U]-[\mathrm{CU}, U]\}, \\
\overrightarrow{\mathbf{s}} & =\inf _{\|U\|_{p+1}=\overrightarrow{\mathbf{1}}}\{[\nabla U, \nabla U]\},
\end{aligned}
$$

where $\||U|\|_{p+1}=\overrightarrow{\mathbf{1}}$ means that $\|u\|_{p+1}=1$ and $\|v\|_{p+1}=1$, and whether $u=0$ then $\|v\|_{p+1}=1$, also, or $v=0$ then $\|u\|_{p+1}=1, U=(u, v) \in \mathbf{L}^{p+1}(\Omega)$ and $p=(n+2) /(n-2)$.

It is important to note that $U>\Theta$ in (3.6) because, in other case, we replace $U$ by $|U|$.

Now we have our main theorem.

THEOREM 3.1. Suppose that

(a) $0<\lambda$ and $\gamma<\lambda_{1}$;

(b) $\delta, \theta \geq 0$;

(c) $\operatorname{det}\left(\lambda_{1} \mathbf{I}-\mathbf{A}\right)>0$;

(d) $\boldsymbol{\lambda}+\boldsymbol{\delta}$ and $\gamma+\theta$ less than $\lambda_{1}$.

Then the problem

$$
-\vec{\Delta} U=\mathbf{A} U+U^{p}, \quad U>\Theta
$$

on $\Omega$ and $U=\Theta$ on $\partial \Omega$, where $p=(n+2) /(n-2), n \geq 4$, has a weak solution.

Proof. Here we follow similar arguments to the one used in [4] for the scalar case. Let $\left\{U_{n}\right\} \subset \mathbf{H}_{0}^{1}(\Omega)$ be a minimizing sequence of $\overrightarrow{\mathbf{S}}_{\mathrm{C}}$. Then

$$
\left[\nabla U_{n}, \nabla U_{n}\right]-\left[\mathrm{CU}_{n}, U_{n}\right]=\overrightarrow{\mathbf{S}}_{\mathrm{C}}+\overrightarrow{o(1)}
$$

as $n \rightarrow \infty$. 
234 A note on the variational structure

Since $\left\|\left|U_{n}\right|\right\|_{p+1}=\overrightarrow{\mathbf{1}}$ for all $n$, then $\left\{U_{n}\right\}$ is bounded in $\mathbf{L}^{2}(\Omega)$ and from (3.8), we conclude that $\left\{U_{n}\right\}$ is bounded in $\mathbf{H}_{0}^{1}(\Omega)$. So, we see that there exists $U \in \mathbf{H}_{0}^{1}(\Omega)$ and $\||U|\|_{p+1} \leq \overrightarrow{\mathbf{1}}$ such that

(a) $U_{n} \rightarrow U$ in $\mathbf{H}_{0}^{1}(\Omega)$;

(b) $U_{n} \rightarrow U$ in $\mathbf{L}^{2}(\Omega)$;

(c) $U_{n} \rightarrow U$ almost everywhere.

Let $V_{n}=U_{n}-U$, then $V_{n} \rightarrow \Theta$ in $\mathbf{H}_{0}^{1}(\Omega), V_{n} \rightarrow \Theta$ in $\mathbf{L}^{2}(\Omega)$, and $V_{n} \rightarrow \Theta$ a.e.

Since $\mathbf{H}_{0}^{1}(\Omega) \hookrightarrow \mathbf{L}^{p+1}(\Omega),\left\|\left|U_{n}\right|\right\|_{p+1}=\overrightarrow{\mathbf{1}}$, and $\overrightarrow{\mathbf{S}} *\|X \mid\|_{p+1}^{2} \leq[\nabla X, \nabla X]$, for all $X \in \mathbf{H}_{0}^{1}(\Omega)$, we conclude, using (3.8), that

$$
\left[\mathrm{C} U_{n}, U_{n}\right]>\overrightarrow{\mathbf{S}}-\overrightarrow{\mathbf{S}}_{\mathrm{C}}
$$

as $n \rightarrow \infty$.

A direct calculation shows that $\overrightarrow{\mathrm{S}}_{\mathrm{C}} \leq \overrightarrow{\mathrm{S}}_{\mathrm{D}}$, where

$$
\mathbf{D}=\left(\begin{array}{cc}
\lambda+\delta & 0 \\
0 & \gamma+\theta
\end{array}\right)
$$

Now, by hypothesis (a) and (b), we see that $\lambda+\delta$ and $\gamma+\theta$ are greater than zero, then $\overrightarrow{\mathbf{S}}_{\mathrm{C}} \leq \overrightarrow{\mathrm{S}}_{\mathrm{D}}$. Also, as in [4, Lemma 1.1], since $n \geq 4$, then $\overrightarrow{\mathbf{S}}_{\mathrm{D}}<\overrightarrow{\mathbf{S}}$. Then, by (3.9), we affirm that $U \neq \Theta$.

Using (3.8), we obtain

$$
[\nabla U, \nabla U]+\left[\nabla V_{n}, \nabla V_{n}\right]-[\mathrm{CU}, U]=\overrightarrow{\mathrm{S}}_{\mathrm{C}}+\overrightarrow{\mathrm{o}(1)} .
$$

A well-known result, due to Brézis and Lieb [3], tells us that

$$
\left\|\left|U+V_{n}\right|\right\|_{p+1}^{p+1}=\||U|\|_{p+1}^{p+1}+\left\|\left|V_{n}\right|\right\|_{p+1}^{p+1}+\overrightarrow{o(1)},
$$

and therefore

$$
\overrightarrow{\mathbf{1}}=\||U|\|_{p+1}^{p+1}+\left\|\left|V_{n}\right|\right\|_{p+1}^{p+1}+\overrightarrow{o(1)} .
$$

So we get

$$
\overrightarrow{1} \leq\||U|\|_{p+1}^{2}+\left\|\left|V_{n}\right|\right\|_{p+1}^{2}+\overrightarrow{o(1)} .
$$

By (3.14) and since $\overrightarrow{\mathbf{S}} *\||X|\|_{p+1}^{2} \leq[\nabla X, \nabla X]$ for all $X \in \mathbf{H}_{0}^{1}(\Omega)$, we have

$$
\overrightarrow{\mathbf{S}} \leq \vec{S} *\||U|\|_{p+1}^{2}+\left[\nabla V_{n}, \nabla V_{n}\right]+\overrightarrow{o(\mathbf{1})} .
$$


Now, since $\overrightarrow{\mathbf{S}}-\overrightarrow{\mathbf{S}}_{\mathbf{C}}>\Theta$ and $\||U|\|_{p+1}^{2} \leq \overrightarrow{\mathbf{1}}$, we use (3.11) and (3.15) and we get

$$
\begin{aligned}
{[\nabla U, \nabla U]-[\mathbf{C U}, U] } & =\overrightarrow{\mathbf{S}}_{\mathrm{C}}-\left[\nabla V_{n}, \nabla V_{n}\right]+\overrightarrow{o(\mathbf{1})} \\
& \leq \overrightarrow{\mathbf{S}}_{\mathrm{C}}+\vec{S} *\||U|\|_{p+1}^{2}-\vec{S}+\overrightarrow{o(\mathbf{1})} \\
& \leq \overrightarrow{\mathbf{S}}_{\mathrm{C}} *\||U|\|_{p+1}^{2} .
\end{aligned}
$$

Inequality (3.16) states that $\overrightarrow{\mathbf{S}}_{\mathrm{C}}$ is really achieved. Now we will use a Lagrange multiplier argument. Let

$$
\begin{gathered}
\mathbf{f}(V)=\frac{1}{2}[\nabla V, \nabla V]-\frac{1}{2}[\mathrm{CV}, V], \\
\mathbf{h}(V)=\||V|\|_{p+1}^{p+1} .
\end{gathered}
$$

A direct calculation shows that for all $V, \Phi \in \mathbf{H}_{0}^{1}(\Omega)$,

$$
\begin{aligned}
\mathbf{f}^{\prime}(V)(\Phi) & =[\nabla V, \nabla \Phi]-\frac{1}{2}\{[\mathbf{C V}, \Phi]+[V, \mathbf{C} \Phi]\}, \\
\mathbf{h}^{\prime}(V)(\Phi) & =(p+1) \int_{\Omega}|V|^{4 /(n-2)} * V * \Phi .
\end{aligned}
$$

Let $U \in \mathbf{H}_{0}^{1}(\Omega)$ be the function for which $\overrightarrow{\mathbf{S}}_{\mathrm{C}}$ is achieved, then there exists a Lagrange multiplier $\mu \in \mathbb{R}^{2}$ such that, for all $\Phi \in \mathbf{H}_{0}^{1}(\Omega)$,

$$
\mathbf{f}^{\prime}(U)(\Phi)=\mu * \mathbf{h}^{\prime}(U)(\Phi) .
$$

In particular, for $\Phi=U$, we get, from (3.18) and (3.19), that

$$
\mu=\frac{1}{p+1}\{[\nabla U, \nabla U]-[\mathbf{C U}, U]\}=\frac{1}{p+1} \overrightarrow{\mathbf{S}}_{\mathrm{C}} .
$$

By (3.18), (3.19), and (3.20) and since we can take $U>\Theta$, we have

$$
[\nabla U, \nabla \Phi]-\frac{1}{2}\{[\mathbf{C U}, \Phi]+[U, \mathbf{C} \Phi]\}=\overrightarrow{\mathbf{S}}_{\mathrm{C}} * \int_{\Omega}|U|^{(n+2) /(n-2)} * \Phi
$$

for all $\Phi \in \mathbf{H}_{0}^{1}(\Omega)$. In particular, if in (3.20) we take $\Phi$ as $\Phi=(\varphi, \varphi)$, (3.21) turns out

$$
[\nabla U, \nabla \Phi]-[\mathbf{A} U, \Phi]=\overrightarrow{\mathbf{S}}_{\mathrm{C}} * \int_{\Omega}|U|^{(n+2) /(n-2)} * \Phi
$$


Equality (3.21) tells us that

$$
-\vec{\Delta} U=\mathbf{A} U+\overrightarrow{\mathbf{S}}_{\mathrm{C}} *|U|^{p}
$$

on $\Omega, U=\Theta$ on $\partial \Omega, n \geq 4$, has a nonzero weak solution. Now, we conclude from hypothesis (d), after a direct calculation, that $\overrightarrow{\mathrm{S}}_{\mathrm{C}}>\Theta$.

Finally, from (3.22) and Theorem 2.2, it follows that $\left(\overrightarrow{\mathbf{S}}_{\mathrm{C}}\right)^{1 /(p-1)} * U$ is a nonnegative weak solution of problem (3.7).

Remark 3.2. It is important to note that, for the case $\delta=\theta=0$, hypothesis (d) of Theorem 3.1 is superfluous. In this case, our system is uncoupled and each equation can be handled separately, so we are in the context of [4, Theorem (1.1)].

Regularity of solutions

As in [2], if $\vec{\Delta} U+\mathbf{a}(x) * U=\Theta$ for $\mathbf{a}(x) \in \mathbf{L}^{n / 2}(\Omega), n \geq 3$, then $U \in \mathbf{L}^{r}(\Omega)$ for all $r>0$. Thereafter $U \in \mathbf{C}^{\infty}(\Omega)$. In our case, we are considering $\mathbf{a}(x) *$ $U=\mathbf{A}(U)+|U|^{P}$. Since $U \in \mathbf{L}^{p+1}(\Omega)$, then $\mathbf{a}(x) \in \mathbf{L}^{n / 2}(\Omega)$.

\section{A problem related to (3.7)}

In this section, we deal with the problem

$$
-\vec{\Delta} U=\mathbf{A} U+|U|^{2^{*}-2} * U
$$

on $\Omega, U=\Theta$ on $\partial \Omega$, and $2^{*}=2 n /(n-2), n \geq 4$.

It is clear that weak solutions of (4.1) are the critical points of the functional

$$
\mathbf{J}(U)=\frac{1}{2}[\nabla U, \nabla U]-\frac{1}{2}[\mathrm{CU}, U]-\frac{1}{2^{*}} \int_{\Omega}|U|^{2^{*}} .
$$

Our main tool is the following theorem.

THeOREM 4.1. For all $\overrightarrow{\mathbf{c}} \in \mathbb{R}^{2}$ such that $\Theta<\overrightarrow{\mathbf{c}}<(1 / n) \overrightarrow{\mathbf{S}}^{n / 2}, n \geq 4$, the functional $\mathbf{J}$ satisfies the Palais-Smale condition on $\overrightarrow{\mathbf{c}}$.

Proof. Let $\left\{U_{i}\right\} \in \mathbf{H}_{0}^{1}(\Omega)$ such that $\mathbf{J}\left(U_{i}\right) \rightarrow \overrightarrow{\mathbf{c}}$ and $\mathbf{J}^{\prime}\left(U_{i}\right) \rightarrow \Theta$, as $i \rightarrow \infty$. This is

$$
\frac{1}{2}\left[\nabla U_{i}, \nabla U_{i}\right]-\frac{1}{2}\left[\mathrm{CU}_{i}, U_{i}\right]-\frac{1}{2^{*}} \int_{\Omega}\left|U_{i}\right|^{2^{*}}-\overrightarrow{\mathbf{c}}=\overrightarrow{o(\mathbf{1})},
$$




$$
\left[\nabla U_{i}, \nabla \Phi\right]-\frac{1}{2}\left\{\left[\mathrm{CU}_{i}, \Phi\right]+\left[U_{i}, \mathbf{C} \Phi\right]\right\}-\left[\left|U_{i}\right|^{2^{*}-2} * U_{i}, \Phi\right]=\overrightarrow{o(\mathbf{1})}
$$

as $i \rightarrow \infty$, for all $U, \Phi \in \mathbf{H}_{0}^{1}(\Omega)$. In (4.4), we put $U=\Phi=U_{i}$ and we get

$$
\left[\nabla U_{i}, \nabla U_{i}\right]-\left[\mathrm{CU}_{i}, U_{i}\right]-\int_{\Omega}\left|U_{i}\right|^{2^{*}}=\overrightarrow{o(\mathbf{1})}
$$

as $i \rightarrow \infty$. Then, the left-hand side of (4.3) minus the left-hand side of (4.5) produces

$$
\int_{\Omega}\left|U_{i}\right|^{2^{*}}=n \overrightarrow{\mathbf{c}}+\overrightarrow{o(\mathbf{1})}
$$

as $i \rightarrow \infty$.

From (4.6), we conclude that $\left\|U_{i}\right\|_{\mathbf{L}^{*}(\Omega)}$ is bounded. Now, since $\mathbf{L}^{2^{*}}(\Omega)$ $\hookrightarrow \mathbf{L}^{2}(\Omega)$ is continuous, then $\left\{U_{i}\right\}$ is bounded on $\mathbf{L}^{2}(\Omega)$; by (4.5) we see that $\left\{U_{i}\right\}$ is bounded on $\mathbf{H}_{0}^{1}(\Omega)$ as well. Therefore, let $U_{0} \in \mathbf{H}_{0}^{1}(\Omega)$ such that

$$
\begin{gathered}
U_{i} \rightarrow U_{0} \quad \text { in } \mathbf{H}_{0}^{1}(\Omega), \\
U_{i} \longrightarrow U_{0} \quad \text { in } \mathbf{L}^{r}(\Omega), 1 \leq r<2^{*},
\end{gathered}
$$

also, $U_{i} \rightarrow U_{0}$ a.e. Now, for all $\Phi \in \mathbf{H}_{0}^{1}(\Omega)$,

$$
\begin{aligned}
\left(\mathbf{J}^{\prime}\left(U_{0}\right)\right. & \left.-\mathbf{J}^{\prime}\left(U_{i}\right)\right)(\Phi) \\
= & {\left[\nabla\left(U_{0}-U_{i}\right), \nabla \Phi\right] } \\
& -\frac{1}{2}\left\{\left[\mathbf{C}\left(U_{0}-U_{i}\right), \Phi\right]+\left[U_{0}-U_{i}, \mathbf{C} \Phi\right]\right\} \\
& +\int_{\Omega}\left(\left|U_{i}\right|^{4 /(n-2)} * U_{i}-\left|U_{0}\right|^{4 /(n-2)} * U_{0}\right) * \Phi .
\end{aligned}
$$

From (4.7a), we see that

$$
\left[\nabla\left(U_{0}-U_{i}\right), \nabla \Phi\right]-\frac{1}{2}\left\{\left[\mathbf{C}\left(U_{0}-U_{i}\right), \Phi\right]+\left[U_{0}-U_{i}, \mathbf{C} \Phi\right]\right\} \rightarrow \Theta
$$

as $i \rightarrow \infty$. Also, from $(4.7 \mathrm{~b})$, we have

$$
\int_{\Omega}\left(\left|U_{i}\right|^{4 /(n-2)} * U_{i}-\left|U_{0}\right|^{4 /(n-2)} * U_{0}\right) * \Phi \longrightarrow \Theta
$$


238 A note on the variational structure

as $i \rightarrow \infty$. So, from (4.8), we conclude that

$$
\left(\mathbf{J}^{\prime}\left(U_{0}\right)-\mathbf{J}^{\prime}\left(U_{i}\right)\right)(\Phi) \longrightarrow \Theta
$$

as $i \rightarrow \infty$. Then, by using the foregoing convergence and our hypothesis $\mathbf{J}^{\prime}\left(U_{i}\right) \rightarrow \Theta$, we conclude that, for all $\Phi \in \mathbf{H}_{0}^{1}(\Omega), \mathbf{J}^{\prime}\left(U_{0}\right)(\Phi)=\Theta$ and therefore

$$
\mathbf{J}^{\prime}\left(U_{0}\right)\left(U_{0}\right)=\left[\nabla U_{0}, \nabla U_{0}\right]-\left[\mathbf{C} U_{0}, U_{0}\right]-\int_{\Omega}\left|U_{0}\right|^{p+1}=0 .
$$

Let $V_{i}=U_{i}-U_{0}$, then

$$
\begin{aligned}
\overrightarrow{o(\mathbf{1})}=\mathbf{J}^{\prime}\left(U_{i}\right)\left(V_{i}\right)= & {\left[\nabla V_{i}, \nabla V_{i}\right]+\left[\nabla U_{0}, \nabla V_{i}\right] } \\
& -\frac{1}{2}\left\{\left[\mathbf{C} U_{i}, V_{i}\right]+\left[U_{i}, \mathbf{C} V_{i}\right]\right\} \\
& -\int_{\Omega}\left|U_{i}\right|^{4 /(n-2)} * U_{i} * V_{i}
\end{aligned}
$$

as $i \rightarrow \infty$.

From (4.7a), (4.7b), (4.13), and identity (3.12), we see that, as $i \rightarrow \infty$,

$$
\begin{aligned}
{\left[\nabla V_{i}, \nabla V_{i}\right]=} & \int_{\Omega}\left|U_{i}\right|^{4 /(n-2)} * U_{i} * V_{i}+\overrightarrow{o(\mathbf{1})} \\
= & \int_{\Omega}\left|U_{0}+V_{i}\right|^{4 /(n-2)} *\left(U_{0}+V_{i}\right) *\left(U_{0}+V_{i}-U_{0}\right)+\overrightarrow{o(\mathbf{1})} \\
= & \int_{\Omega}\left|U_{0}+V_{i}\right|^{p+1}-\int_{\Omega}\left|U_{0}+V_{i}\right|^{4 /(n-2)} *\left(U_{0}+V_{i}\right) * U_{0}+\overrightarrow{o(\mathbf{1})} \\
= & \left\|U_{0}\right\|_{p+1}^{p+1}+\left\|V_{i}\right\|_{p+1}^{p+1} \\
& -\int_{\Omega}\left|U_{0}+V_{i}\right|^{4 /(n-2)} *\left(U_{0}+V_{i}\right) * U_{0}+\overrightarrow{o(\mathbf{1})} .
\end{aligned}
$$

Since $V_{i} \rightarrow \Theta$ in $\mathbf{L}^{r}(\Omega)$, for $1 \leq r<2^{*}$, we get

$$
\left[\nabla V_{i}, \nabla V_{i}\right]=\left\|V_{i}\right\|_{2^{*}}^{2^{*}}+\overrightarrow{o(\mathbf{1})} .
$$

From (4.2) and (4.5), we deduce that

$$
\mathbf{J}\left(U_{i}\right)=\frac{1}{n}\left\{\left[\nabla U_{i}, \nabla U_{i}\right]-\left[\mathbf{C U}_{i}, U_{i}\right]\right\}+\overrightarrow{o(\mathbf{1})}
$$

as $i \rightarrow \infty$. 
Now, since $V_{i} \rightarrow \Theta$ in $\mathbf{L}^{2}(\Omega)$ and $V_{i} \rightarrow \Theta$ in $\mathbf{H}_{0}^{1}(\Omega)$, from (4.16), we obtain

$$
\mathbf{J}\left(U_{i}\right)=\frac{1}{n}\left\{\left[\nabla U_{0}, \nabla U_{0}\right]-\left[\mathrm{CU}_{0}, U_{0}\right]\right\}+\frac{1}{n}\left[\nabla V_{i}, \nabla V_{i}\right]+\overrightarrow{o(\mathbf{1})}
$$

as $i \rightarrow \infty$.

Now, from (4.12), we see that $\left[\nabla U_{0}, \nabla U_{0}\right]-\left[C U_{0}, U_{0}\right] \geq \Theta$, then, by (4.17), we have

$$
\left[\nabla V_{i}, \nabla V_{i}\right] \leq n \mathbf{J}\left(U_{i}\right)+\overrightarrow{o(1)}
$$

as $i \rightarrow \infty$.

The foregoing inequality, united with our hypothesis $\mathbf{J}\left(U_{i}\right) \rightarrow \overrightarrow{\mathbf{c}} \in(\Theta$, $\left.(1 / n) \overrightarrow{\mathbf{S}}^{n / 2}\right)$, produces

$$
\left[\nabla V_{i}, \nabla V_{i}\right] \leq \text { const }<\overrightarrow{\mathbf{S}}^{n / 2}
$$

for all $i$ large enough.

It is important to note that $[\nabla \Phi, \nabla \Phi]=\|\Phi\|_{\mathbf{H}_{0}^{1}(\Omega)}^{2}$ and $\Phi \in \mathbf{H}_{0}^{1}(\Omega)$. Now, since $\mathbf{H}_{0}^{1}(\Omega) \hookrightarrow \mathbf{L}^{2^{*}}(\Omega)$ and

$$
\sqrt{\overrightarrow{\mathbf{S}}}\|\Phi\|_{\mathbf{L}^{2^{*}}(\Omega)} \leq\|\Phi\|_{\mathbf{H}_{0}^{1}(\Omega)}
$$

we deduce from (4.15) that

$$
\left\|V_{i}\right\|_{\mathbf{H}_{0}^{1}(\Omega)}^{2}\left(\overrightarrow{\mathbf{S}}^{2^{*} / 2}-\left\|V_{i}\right\|_{\mathbf{H}_{0}^{1}(\Omega)}^{2^{*}-2}\right) \leq \overrightarrow{o(\mathbf{1})}
$$

as $i \rightarrow \infty$. Now, from (4.19) and since $n \geq 4$, we deduce that $\overrightarrow{\mathbf{S}}^{2^{*} / 2}-\left\|V_{i}\right\|_{\mathbf{H}_{0}^{1}(\Omega)}^{2^{*}-2}$ is greater than a positive constant for $i$ large enough. Then from (4.21), we conclude that $V_{i} \rightarrow \Theta$.

Now, we are ready for the following theorem.

\section{THEOREM 4.2. Suppose that}

(e) $\boldsymbol{\lambda}+\boldsymbol{\delta}, \gamma+\theta<\lambda_{1}$

(f) $\lambda+\delta, \gamma+\theta>0$

Then problem (4.1) has at least a nonzero solution.

Proof. First, we conclude, from hypothesis (e), that $\overrightarrow{\mathbf{S}}_{C}>\Theta$, then for all $U_{0}=\left(u_{0}, v_{0}\right) \in \mathbf{H}_{0}^{1}(\Omega),\left\|U_{0}\right\|_{\mathbf{L}^{2^{*}}(\Omega)}^{2^{*}}=\overrightarrow{\mathbf{1}}$, we have

$$
\mathbf{M}=\left[\nabla U_{0}, \nabla U_{0}\right]-\left[\mathbf{C U}_{0}, U_{0}\right]>\overrightarrow{\mathbf{S}}_{C}>\Theta .
$$


Now, we show that, for all $U_{0}=\left(u_{0}, v_{0}\right) \in \mathbf{H}_{0}^{1}(\Omega)$ and $\left\|U_{0}\right\|_{\mathrm{L}^{2^{*}}(\Omega)}^{2^{*}}=\overrightarrow{\mathbf{1}}$,

$$
\frac{1}{n} \overrightarrow{\mathbf{S}}_{C}^{n / 2} \leq \sup _{\overrightarrow{\mathbf{t}}>\Theta} \mathbf{J}\left(\overrightarrow{\mathbf{t}} * U_{0}\right) \leq \frac{1}{n} \overrightarrow{\mathbf{S}}^{1 / n}
$$

In fact, let

$$
\psi(\mathbf{t})=\mathbf{J}\left(\overrightarrow{\mathbf{t}} * U_{0}\right)=\frac{1}{2} \mathbf{M} * \overrightarrow{\mathbf{t}}^{2}-\frac{1}{2^{*}} \overrightarrow{\mathbf{t}}^{2^{*}}, \quad \overrightarrow{\mathbf{t}} \in \mathbb{R}^{2} .
$$

It is clear that $\psi(\overrightarrow{\mathbf{t}})>\Theta$, for $\overrightarrow{\mathbf{t}}>\Theta$ small enough and $\lim _{t \rightarrow \infty} \psi(\overrightarrow{\mathbf{t}}) \rightarrow-\infty$ . Now, by a direct calculation, we see that, for $\overrightarrow{\mathbf{t}}=\mathbf{M}^{(n-2) / 4}, \psi(\overrightarrow{\mathbf{t}})$ reaches its maximum value. Then, from (4.22), we get

$$
\sup _{t>\Theta} \psi(\overrightarrow{\mathfrak{t}})=\frac{1}{n} \mathbf{M}^{n / 2}>\frac{1}{n} \overrightarrow{\mathbf{S}}_{C}^{n / 2} .
$$

Finally, from our hypothesis (f), we deduce that $\overrightarrow{\mathbf{S}}_{C}<\overrightarrow{\mathbf{S}}$, then

$$
\sup _{\mathbf{t}>\Theta} \psi(\overrightarrow{\mathbf{t}})<\frac{1}{n} \overrightarrow{\mathbf{S}}^{n / 2}
$$

Therefore, (4.25) and (4.26) produce (4.23). Now, by Theorem 4.1, $\mathbf{J}(\Theta)=$ $\Theta, \psi(\overrightarrow{\mathfrak{t}}) \rightarrow-\infty, \overrightarrow{0}$, and (4.23), we apply the mountain pass lemma of Ambrosetti and Rabinowitz [1], adapted in this case to our functional $\mathbf{J}$, to prove the existence of $\overrightarrow{\mathbf{c}} \in \mathbb{R}^{2}$ such that

$$
\overrightarrow{\mathbf{c}}=\inf \sup \mathbf{J}(U) \in\left(\Theta, \frac{1}{n} \overrightarrow{\mathbf{S}}^{n / 2}\right),
$$

where inf and sup are taken on suitable sets and $\mathbf{J}^{-1}(\overrightarrow{\mathbf{c}}) \neq \Phi$. Therefore, $U \in \mathbf{J}^{-1}(\overrightarrow{\mathbf{c}})$ is a nonzero weak solution of (4.1).

The biharmonic equation

The results of foregoing theorem can be applied to the following class of nonlinear biharmonic equation under Navier-Dirichlet boundary conditions:

$$
\Delta^{2} u=\theta u+|u|^{2^{*}-2} u
$$

on $\Omega, u=\Delta u=0$ on $\partial \Omega$, and $\theta>0$. Indeed, $U=(u, v)$ with $v=-\Delta u$ satisfies the problem

$$
-\vec{\Delta} U=\mathbf{A} U+G(U)
$$


on $\Omega$ and $U=\Theta$ on $\partial \Omega$, where

$$
\mathbf{A}=\left(\begin{array}{ll}
0 & 1 \\
\theta & 0
\end{array}\right), \quad G(U)=\left(|u|^{2^{*}-2} u, 0\right)
$$

As a direct application of Theorem 4.2, we obtain the following theorem.

THEOREM 4.3. Suppose that

(g) $\theta, 1<\lambda_{1}$;

(h) $\theta>0$ (this is a cooperative case).

Then problem (4.28) has a weak solution $u \in H^{3}(\Omega) \cap H_{0}^{1}(\Omega)$ with $\Delta u \in H_{0}^{1}(\Omega)$.

\section{References}

[1] A. Ambrosetti and P. H. Rabinowitz, Dual variational methods in critical point theory and applications, J. Functional Analysis 14 (1973), 349-381.

[2] H. Brézis and T. Kato, Remarks on the Schrödinger operator with singular complex potentials, J. Math. Pures Appl. (9) 58 (1979), no. 2, 137-151.

[3] H. Brézis and E. Lieb, A relation between pointwise convergence of functions and convergence of functionals, Proc. Amer. Math. Soc. 88 (1983), no. 3, 486-490.

[4] H. Brézis and L. Nirenberg, Positive solutions of nonlinear elliptic equations involving critical Sobolev exponents, Comm. Pure Appl. Math. 36 (1983), no. 4, $437-477$.

[5] D. G. Costa and C. A. Magalhães, A variational approach to subquadratic perturbations of elliptic systems, J. Differential Equations 111 (1994), no. 1, 103122.

[6] J. Fleckinger, J. Hernández, and F. de Thélin, On maximum principles and existence of positive solutions for some cooperative elliptic systems, Differential Integral Equations 8 (1995), no. 1, 69-85.

[7] J. López-Gómez and M. Molina-Meyer, The maximum principle for cooperative weakly coupled elliptic systems and some applications, Differential Integral Equations 7 (1994), no. 2, 383-398.

[8] S. Pohozaev, Eigenfunctions of the equation $\Delta u+\lambda f(u)=0$, Soviet Math. Dokl. 6 (1965), 1408-1411, translated from Russian Dokl. Akad. Nauk SSSR, 165 (1965), 33-36.

[9] M. Zuluaga, Nonzero and positive solutions of a superlinear elliptic system, Arch. Math. (Brno) 37 (2001), no. 1, 63-70.

Mario Zuluaga: Departamento de Matemáticas, Universidad Nacional de Colombia, Bogota, Colombia

E-mail address: mzuluaga@matematicas.unal.edu.co 


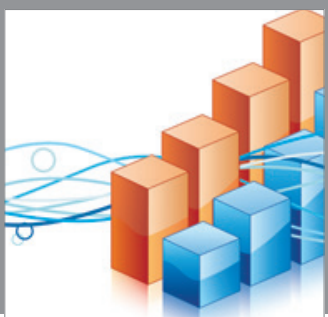

Advances in

Operations Research

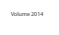

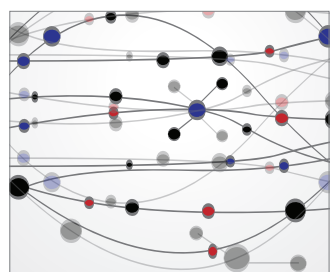

\section{The Scientific} World Journal
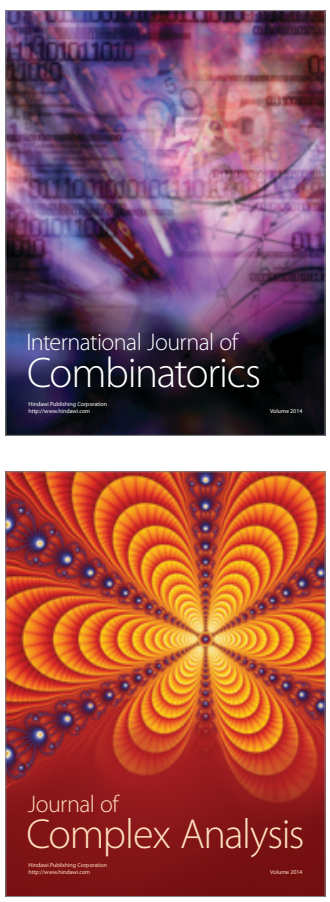

International Journal of

Mathematics and

Mathematical

Sciences
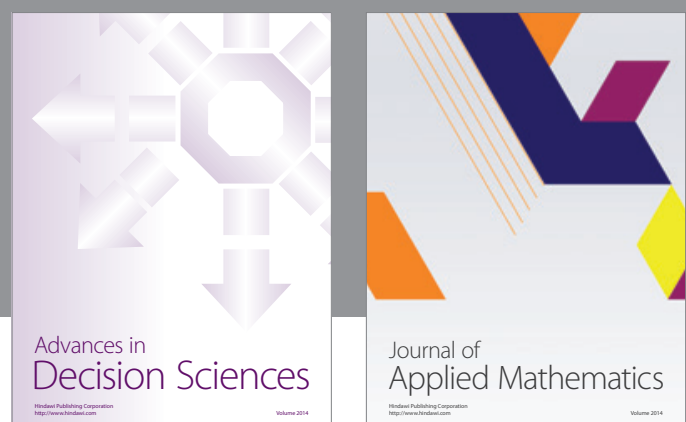

Journal of

Applied Mathematics
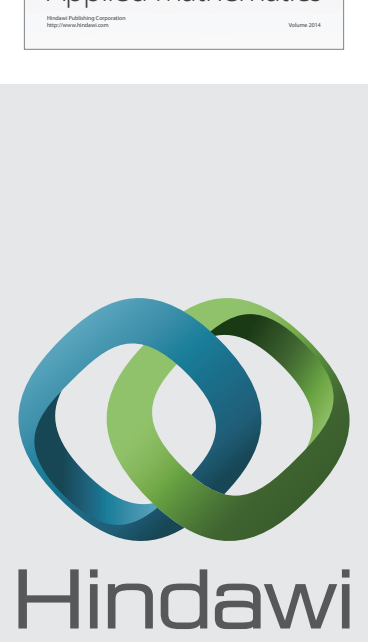

Submit your manuscripts at http://www.hindawi.com
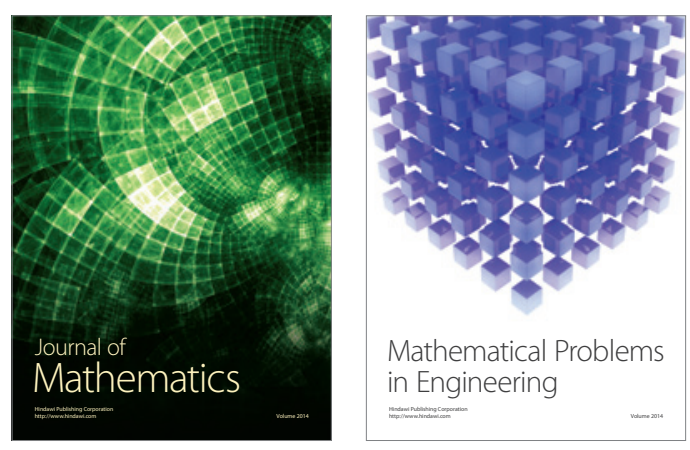

Mathematical Problems in Engineering
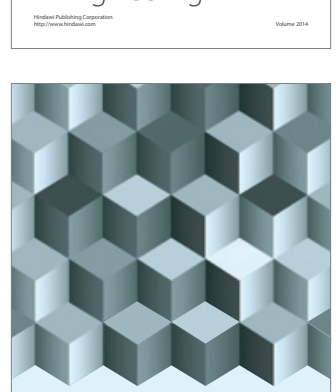

Journal of

Function Spaces
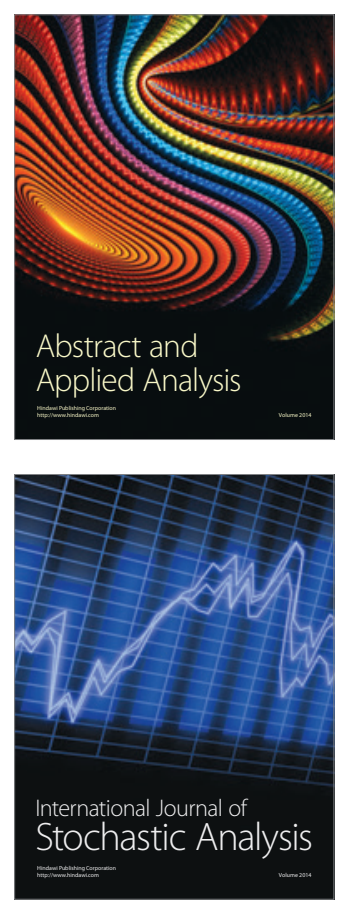

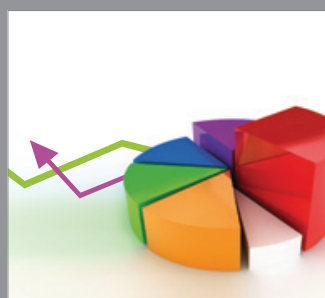

ournal of

Probability and Statistics

Promensencen
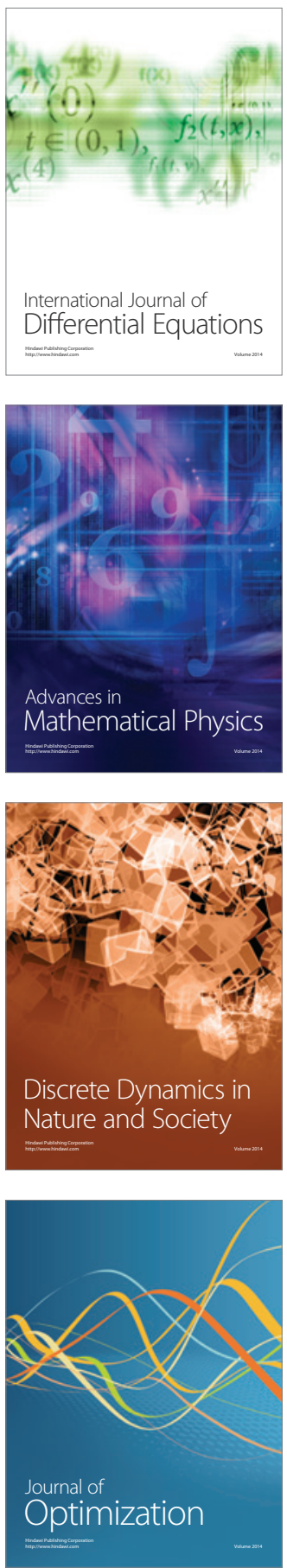